\title{
Aortoileal fistula as a rare cause of bleeding from the gastrointestinal system - Case Report
}

\author{
Wojciech Cios' ${ }^{1}$ Andrzej Prystupa', Anna Nocuń ${ }^{2}$ Kamil Pudło³ , Katarzyna Sołdaj-Bukszyńska' \\ ${ }^{1}$ Department of Internal Medicine, Medical University, Lublin, Poland \\ ${ }^{2}$ Department of Radiology, Medical University, Lublin, Poland \\ ${ }^{3}$ Department of Surgery, Medical University, Lublin, Poland \\ Cios W, Prystupa A, Nocuń A, Pudło K, Sołdaj-Bukszyńska K. Aortoileal fistula as a rare cause of bleeding from the gastrointestinal system \\ - Case Report. J Pre-Clin Clin Res. 2018; 12(4): 126-128. doi: 10.26444/jpccr/100372
}

\section{Abstract}

Introduction. Gastrointestinal bleedings are often a manifestation of ongoing disease processes within the Gl tract. Thanks to widely available endoscopic and imaging examinations, they can be quickly and accurately diagnosed.

Case report. A 68-year-old male patient was admitted to the Department of Internal Diseases due to lower Gl bleedings. The patient's history revealed endovascular aneurysm repair and right coronary artery drug-eluting stent angioplasty. The patient underwent multiple diagnostic procedures. Once the potential site of bleeding had been found and emergency exploratory laparotomy performed, visualization was enabled of the location of an aortoileal fistula caused by a prosthesis decubitus.

Conclusions. In patients with aortic or aortoileal prostheses and symptoms of acute haemorrhage, an aortoenteric fistula should always be considered. Diagnosis should be based on complementary endoscopic and radiological examinations.

\section{Key words}

endoscopy, fistula, gastrointestinal bleeding, aortoenteric fistula, aortic stentgraft, aortoileal, aortoileal fistula, 99mTclabelled erythrocyte scintigraphy

\section{INTRODUCTION}

GI bleedings are a relatively common manifestation of intestinal diseases observed in clinical practice. About $80 \%$ of bleedings affect the upper GI tract (above the ligament of Treitz) and are predominantly caused by duodenal ulcer, acute haemorrhagic gastropathy, gastric ulcer, Mallory -

Weiss syndrome or cancers. The patient's mortality with symptoms of bleeding occurs in between $6-10 \%$ in upper gastroinstestinal bleeding and 4-40\% in lower gastrointestinal bleeding. Bleedings to the lumen of the lower GI tract constitute the remaining 20\% of cases and are most commonly caused by diverticulosis, intestinal inflammations, small and large intestine polyps, vascular malformations, and aortoenteric fistulae [1]. Sometimes a diagnostic laparotomy is also needed [1].

\section{CASE REPORT}

A 68-year-old male patient was admitted to the Department due to lower gastrointestinal (GI) bleeding. Rectal examination revealed a large amount of fresh blood. The patient had been hospitalised three years earlier because of a dissecting aneurism of the abdominal aorta; he underwent aortobifemoral bypass surgery (classic access, laparotomy with a medial incision). Two and a half years prior to admission to our Department, the patient developed symptoms of inferior myocardial infarction, treated with angioplasty with drug-eluting stent (DES) implantation to the right coronary artery. The patient reported epileptic seizures.

For the last six months, the patient had recurring abdominal

Address for correspondence: Wojciech Cios, Department of Internal Medicine, Medical University, Lublin, Poland

E-mail:wojciech.cios@gmail.com

Received: March 2018; Accepted: November 2018 pain with upper and lower GI bleedings and marked anaemia up to $6.1 \mathrm{~g} \% \mathrm{Hb}$. During earlier hospitalisations in the District Hospital the patient underwent three gastroscopies and two colonoscopies which, however, failed to reveal the site of bleeding. Moreover, the small intestine was evaluated using an endoscopic capsule, yet neither the site of bleeding nor fresh intestinal blood were demonstrated.

Since the onset of symptoms, faecal occult blood was found multiple times. Abdominal CT enteroclysis did not reveal any lesions which could be responsible for the symptoms presented; there were no proliferative lesions or abnormalities within the stent-graft placement site.

On admission, the level of haemoglobin was $9.8 \mathrm{mg} /$ dl. During hospitalisation, acute bleeding from the lower GI tract was observed, which resulted in a decrease in $\mathrm{Hb}$ to $6.8 \mathrm{mg} \%$. The patient required transfusions of red-cell concentrate preparations (RCPs) during bleeding - in total, 6 units of RCPs.

The patient was scheduled for emergency colonoscopy, which visualised the final part of the ileum $(18-20 \mathrm{~cm})$, and a small-sized, flat lesion with petechiae of angiomatous nature was detected; argon plasma coagulation was applied.

During abdominal arteriography, the superior mesenteric artery was contrasted, followed by its branches, i.e. middle colic artery, ileocolic artery and the branches to the ileum; no contrast medium extravasation was demonstrated. Subsequently, the patient underwent $99 \mathrm{mTc}$-labelled erythrocyte scintigraphy during bleeding. Single photon emission computed tomography/computed tomography visualised a small and poorly contrasted area of enhanced radioactivity in the ileal projection, on the right abdominal side at the level of the inferior pole of the right kidney (L3) [Fig. 1]. After surgical consultation, the patient was transferred to the Surgical Department for further treatment.

After obtaining informed consent for surgery, the patient was scheduled for exploratory laparotomy. During the 


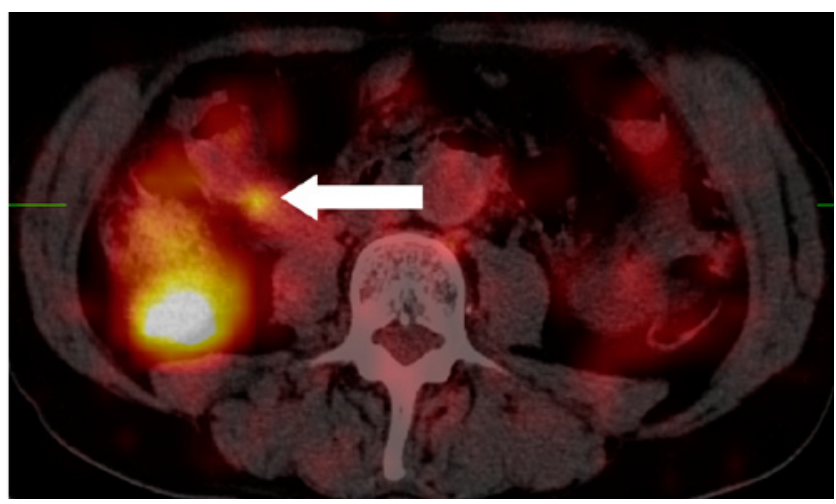

Figure 1. $99 \mathrm{mT}$-labeled red cell SPECT/CT* images of the abdomen with focal activity (arrow) suggesting the site of bleeding in the ileum. *SPECT/CT- single photon emission computed tomography/computed tomography

procedure, the abdominal organs were meticulously assessed and no visible pathologies were found. Numerous adhesions of the small intestine hindered the GI tract evaluation. Once the adhesions were liberated, the entire small intestine was assessed, starting from the ileocaecal valve. About one metre from the ligament of Treitz, the adhesion of the small intestine wall with the abdominal aorta was visualised. After its liberation through an opening in the abdominal aorta wall, the aortobifemoral prosthesis wall was exposed (Fig. 2). No active bleeding from the region of the fistula was found intraoperatively. A fragment of the small intestine with the fistula was removed (Fig. 3, 4). and side-to-side bowel anastomosis performed. The aortic walls were secured with a thrombin-soaked sponge. After completion of surgical treatment and convalescence, the patient was transferred to the Department of Infectious Diseases due to Clostridium difficile infection.

After the treatment, the patient was discharged home in good condition with recommendation for the next, urgent specialistic treatment in the Vascular Surgery Ward. After the above-described treatment, the authors receive the residual information that the patient had experienced an episode of bleeding, but have no information about course of further treatment.

\section{DISCUSSION}

Aortoenteric fistulas are classified into primary and secondary fistulae. The former are caused by disease processes in the immediate vicinity of the aorta and intestines, or are directly associated with these organs, e.g. abdominal aorta aneurysm, peptic ulcer or neoplastic infiltration. The latter can be a complication of increasingly common procedures performed to repair aortic diseases, e.g. implantation of aortic prostheses for the treatment of ruptured aneurysms, or those at risk of rupturing [2].

The first alarming symptoms can occur at different postoperative times, ranging from several to 14 years; on average, about three years after the primary vessel repair [3]. In the presented case, it was about two and a half years after prosthesis implantation. The prodromal symptoms include minor, self-limiting bleedings, which can result in sudden and abundant haemorrhage leading to shock or death.

The condition discussed resulted from adhesions of the prosthesis to the adjacent intestinal wall and the formation

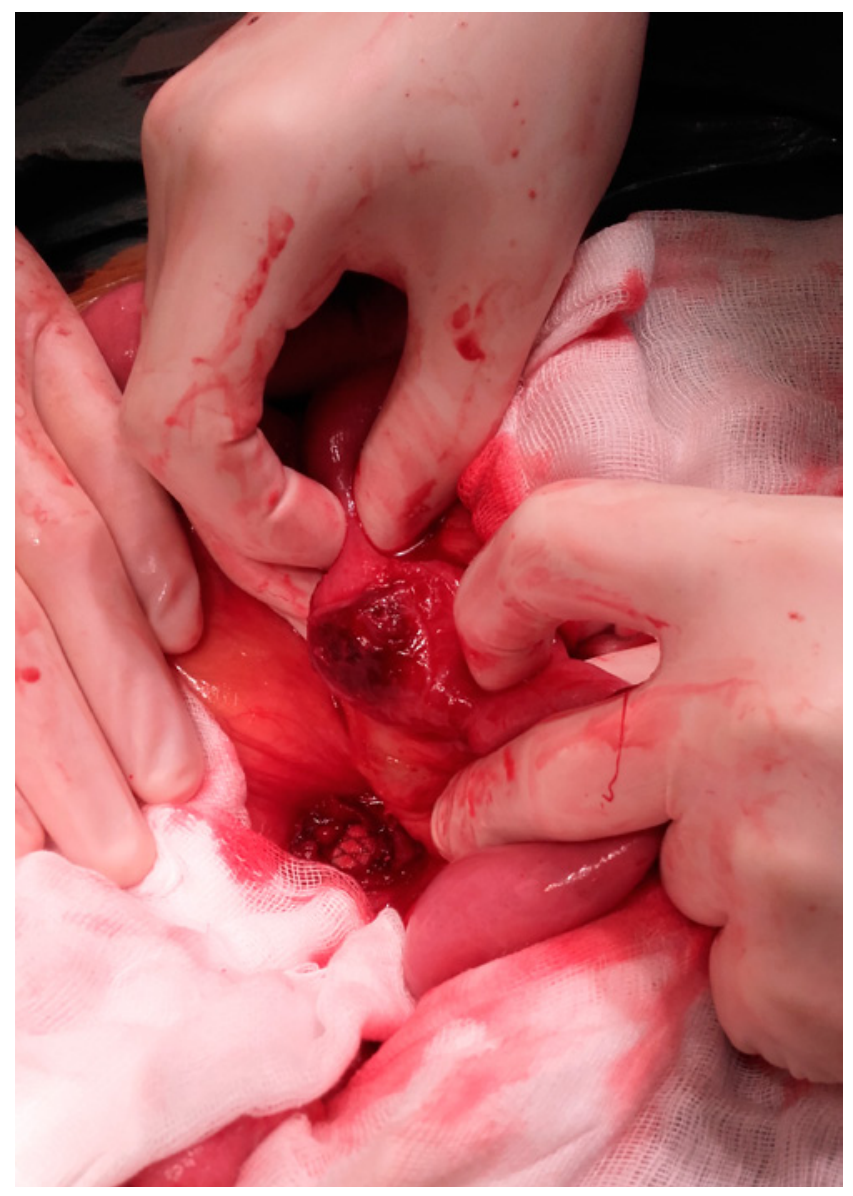

Figure 2. The view of the operating field

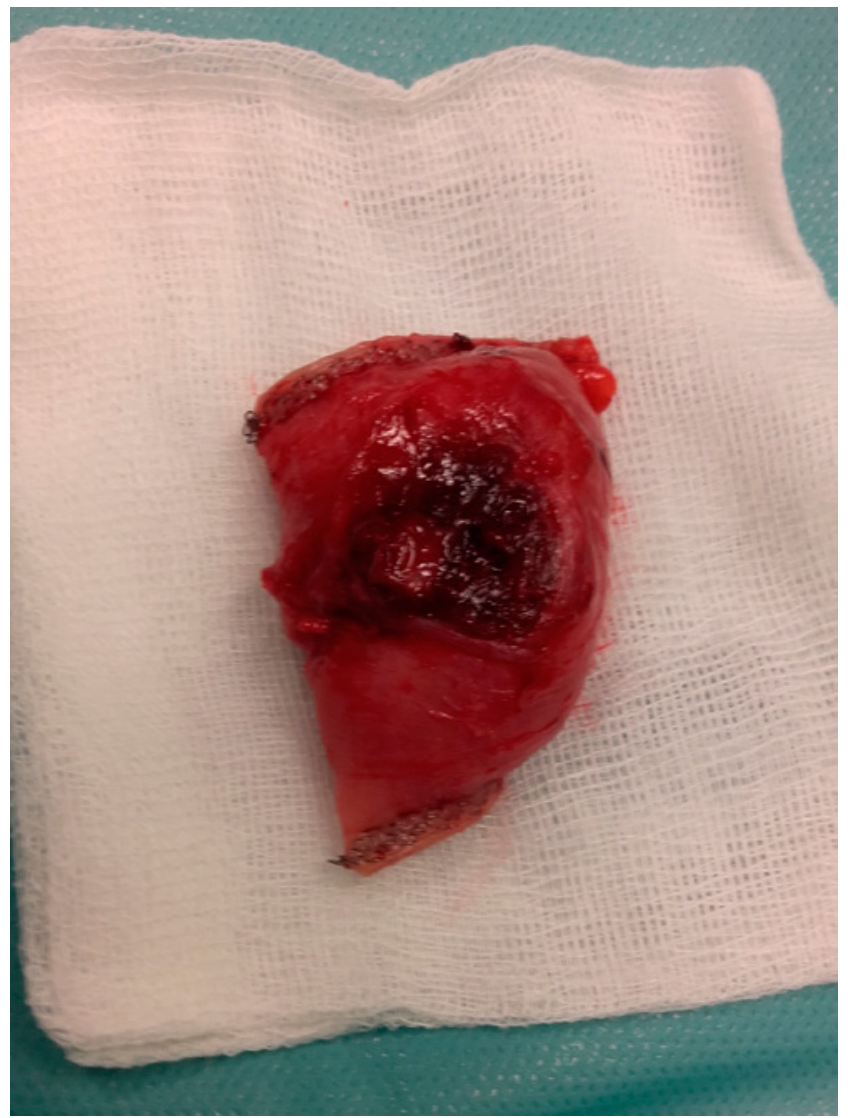

Figure 3. The dissected part of the small intestine 


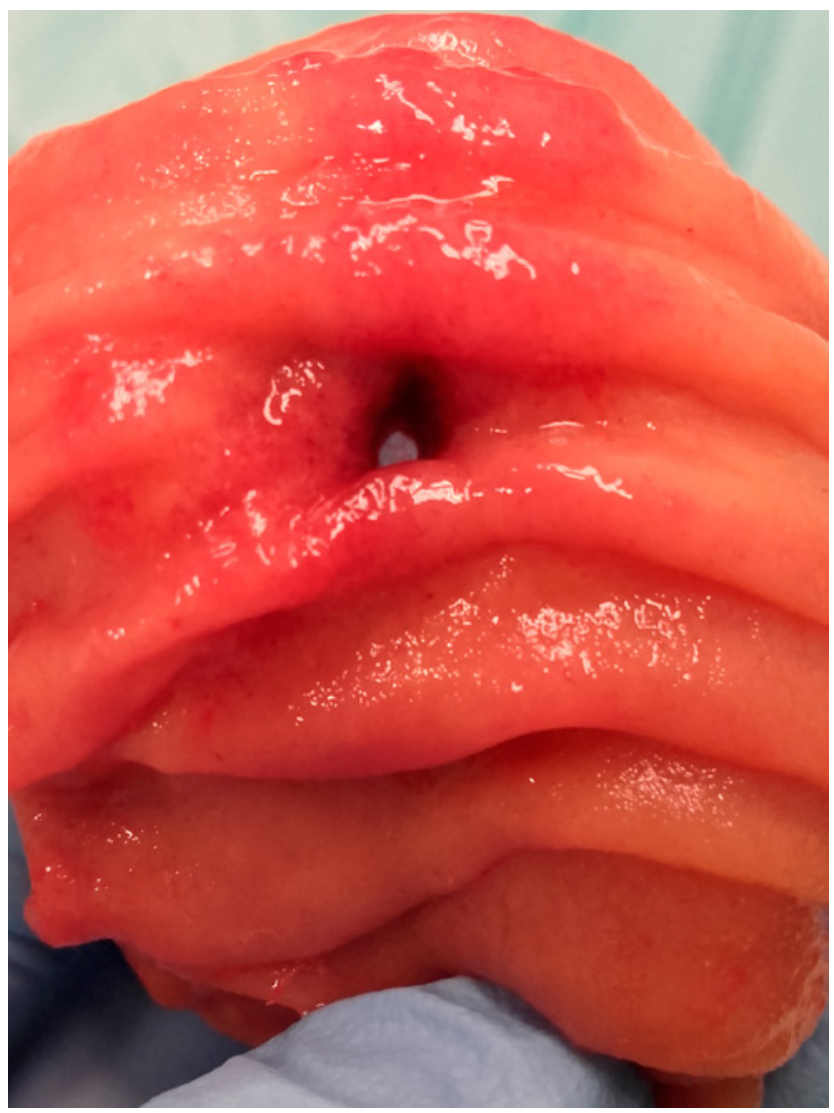

Figure 4. The dissected part of the small intestine, the internal view of the specimen

of decubitus ulcer that penetrated the wall, leading to communication between the lumens of both organs.

Almost $78 \%$ of cases of aortoenteric fistulae involve a connection between the aorta and duodenum; ileoaortic fistulae constitute only $4 \%$ of all types of connections. The time to diagnosis ranges from 14-37 days [4].

GI endoscopy is the basic diagnostic examination, which in the presented case (a total of 8 endoscopies) failed to determine the source of bleeding. Moreover, arteriography was of little use, although it was carried out during active bleeding. Only $99 \mathrm{~m}$ Tc-labelled erythrocyte scintigraphy enabled the location and confirmation of a small, only several-centimetre-big source of bleeding.

When a fistula is suspected, exploratory laparotomy should be promptly performed to detect the source of bleeding. Since the condition of the patient deteriorated, surgical procedure was the quickest and most reliable option to establish the final diagnosis and initiate treatment.

Literature reports describe cases of aorto-colonic fistula repair by replacing the aortic prosthesis with a silver acetatecoated prosthesis. The literature data regarding comparisons of treatment of patients with aortoenteric fistula demonstrate an extremely high intraoperative mortality, even up to $25 \%$; therefore, once an accurate diagnosis has been determined, the treatment of choice is endovascular prosthesis replacement $[5,6]$. The basic method of treatment is removal of the prosthesis in which the infection has developed due to its contact with the intestinal lumen, and subsequent repair of the vascular wall and the intestine.

In patients with aortic or aortoileal prostheses and the symptoms of lower and upper GI tract bleedings, the presence of an aortoenteric fistula should always be considered, even though its incidences are relatively low, whereas the diagnostic procedures should be based on complementary endoscopic and radiological examinations [7].

\section{REFERENCES}

1. Fabiszewski A, Jackowski M. Bleeding from the splenic artery into a pancreatic pseudocyst with a coexistingmicrofistula to the transverse colon as atypical recurrent massive gastrointestinal bleeding. Polish Journal of Surgery 2009; 81(11): 542-549.

2.Pupka A, Skóra J, Pawłowski S, Dawiskiba T, Szyber P. The Surgical treatment of aorto-duodenal fistulas after reconstructive operations of the abdominal aorta. Polish Surgery 2003; 5(2), 83-90.

3. Townsend CM, Beauchamp RD, Evers BM, Mattox KL. Sabiston Textbook of Surgery, Ed 18, part IV. In: Popiela T, editor. Acute bleeding from the gastrointestinal tract. Wrocław 2013, p. 89.

4. Pupka A, Szyber PP, Janczak D, Pawłowski S, Szyber P. The treatment of abdominal aortic aneurysms by use of endovascular prosthesis and classic vascular prosthesis. http://www.dbc.wroc.pl/Content/1976/302_ Pupk2.pdf (Access 08.03.2018)

5. Gnus J, Ferenc S, Kościelna M, Paprocka-Borowicz M, Dawidczyk P, Dziewiszek M, Witkiewicz W. Secondary Aortoenteric Fistula After Abdominal Aortic Graft Implementation in Our OwnMaterial. Adv Clin Exp Med. 2016; 25(6): 1265-1271.

6. Vaillant JC, Schoell T, Karoui M, Chiche L, Gaudric J, Gibert H, Tresallet C, Koskas F, Hannoun L. Abdominal secondary aorto-enteric fistulae complicating aortic graft replacement: postoperative and long-term outcomes in 32 patients. Med Intensiva. 2013; 37(7): 493-494.

7. Çiçek ÖF, Çiçek MC, Kadiroğulları E, Uzun A, Ulaş M. Successful Treatment of Secondary Aortoenteric Fistula with a Special Graft. Case Reports in Medicine Vol. 2016(2016), Article ID 9874187, http://dx.doi. org/10.1155/2016/98741

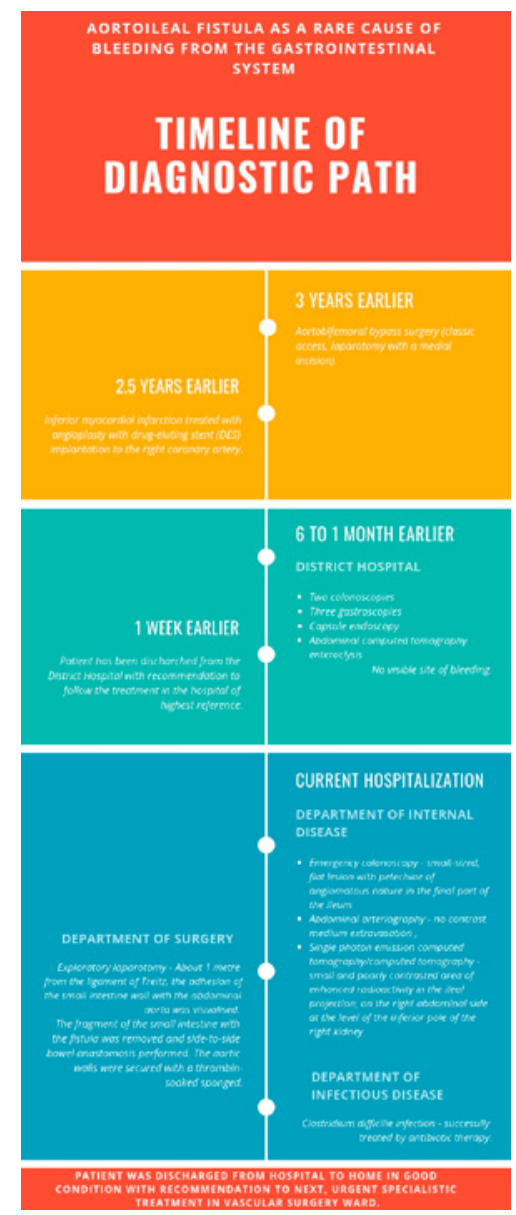

Figure 5. Graphic illustration of the diagnostic path 